\title{
Direct Drive Hand Exoskeleton for Robot-assisted Post Stroke Rehabilitation
}

\author{
Márk Ottó Bauer ${ }^{1}$, Máté Benjámin Vizi ${ }^{2}$, Péter Galambos ${ }^{3}$, \\ and Tibor Szalay ${ }^{4}$
}

\begin{abstract}
${ }^{1,4}$ Department of Manufacturing Science and Engineering, Budapest University of Technology and Economics, Múegyetem rkp. 3. H-1111 Budapest, Hungary, \{bauer.mark, szalay.tibor\}@manuf.bme.hu

${ }^{2}$ Department of Applied Mechanics, Budapest University of Technology and Economics, Múegyetem rkp. 5. H-1111 Budapest, Hungary, vizi@mm.bme.hu ${ }^{3}$ Antal Bejczy Center for Intelligent Robotics, Óbuda University, Bécsi út 96/B. H-1034 Budapest, Hungary, peter.galambos@irob.uni-obuda.hu
\end{abstract}

\begin{abstract}
This article introduces novel rehabilitation hand module development for the physiotherapy of the hand of patients suffering from spastic hemiplegia. Spasm is basically a muscle cramp, it practically involves the sudden, unintended and painful contraction of a muscle or muscle group, which is caused by nerve damage resulting from a stroke. Stroke is the main reason for permanent disability in adulthood, and so the social-and medical care systems require a huge amount of healthcare resources due to the inactivity of the patients concerned. The robotically facilitated rehabilitation assists the physicians in providing repeated therapies of great intensity, and so the patients may enjoy the benefits of rehabilitation, while the therapists may reduce their own workload at the same time. Furthermore, the robotic devices offer an objective and reliable opportunity for tracing and accurately assessing the improvement of the patients' motor skills. This article introduces the electrical-and mechanical design of a therapeutic device and the inverse kinematic and dynamic modules which control this device. The rehabilitation device is capable of moving the thumb, the index-, the middle- and the ring fingers, and allows the rehabilitation of the left-and right hands as well. The device is a completely new design with direct drive approach and several benefits. It has two components: a planar module with serial kinematics of rotational joints with three degrees of freedom (3DoF RRR), and another module with two degrees of freedom (2DoF). The modules integrated load cells, which are built in between each joint to measure the reaction forces. The 3DoF finger moves the index, the middle and the ring fingers, using a load distributor placed above the fingers. The finger orthoses are connected to the load distributor via magnets. The 2DoF finger moves the thumb performing the opening/closing along the plane tilted in two angles.
\end{abstract}

Keywords: Rehabilitation robotics, Hand rehabilitation, Hand exoskeleton, Wearable structure, Force control 


\section{Introduction}

Stroke is one of the critical causes of disability in cognition or movement. It often results in a combination of cognitive, motor and sensory impairments. Now, it has become one of the main diseases threatening human health and the healthy survival $^{1}$. One of the most common impairment of stroke is the hemiparesis, which results in dyskinesia of some parts of the body. It reflects not only in the upper and lower limbs, but also in the loss of motor function of the hand. Hand rehabilitation requires repetitive task exercises, where a task is divided into several movements and patients need to practice those movements to improve their hand strength, motion accuracy, and range of motion, but high cost of traditional treatments are often prevent patients from spending enough time on the necessary rehabilitation. Few studies show that repetitive movements with robotic assistance can significantly improve the hand motor functions of patients [1]. Hand is one of the most important limb of humans, and the rehabilitation of hand motor function can be assisted by exoskeleton robots, because the application of robotics and related technologies has greatly promoted the development of clinical techniques. At the last 10-20 years many dexterous and advanced mechanisms of hand exoskeletons have been developed and tested. But not all of them are developed for hand rehabilitation. For example some of them are designed as force-feedback devices and some others are designed for master-slave gripping systems. Hand rehabilitation exoskeleton devices are still seeking to achieve key features such as bi-directional actuation, low cost, low complexity, compactness, portability, safe interaction between human and machine, and simple and reliable control.

The robot-assisted hand/finger rehabilitation is recently in the focus of researchers, so it has a broad literature. There are several approaches to solve the difficulties appearing in the case of hand and finger movement, the main constraints are the available space for the robot and the relatively large forces required to move the spastic fingers. The most common approaches considering the actuator types are the pneumatic gloves and the electric motors with indirect drive through a wire/cable system. Also worth to mention a new direction in the this field, rapid development of material sciences results in new possibilities, shape memory alloys can be used as the active actuators [2] in hand exoskeletons. Pneumatic gloves use air-pressurized chambers on the palmar side of the hand for finger extensions; if these are the only actuators, then only the finger extension is controlled by the robot $[1,3]$ (see Figure 1). The finger flexion can be achieved by a supplemented motor-cable system [4] or by pre-loaded passive springs [5] on the dorsal side of the hand. The other common approach uses electric motors, the produced torques are applied on the finger joints through a cable/wire system [6, 7] (see Figure 1). Separate motors are necessary for flexion and extension because of the soft cables transmitting the force, so two motors must be used for each controlled joints. These solutions result in a relatively thin exoskeletons [8,9] or gloves [10,11], but the overall required space is large $[12,13]$ due the high number of motors. All of mentioned studies threat each of the five

1 https://www.healthline.com/health/top-10-deadliest-diseases 


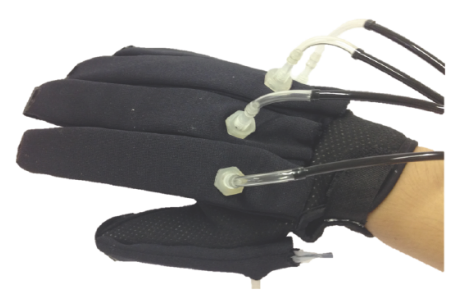

Figure 1

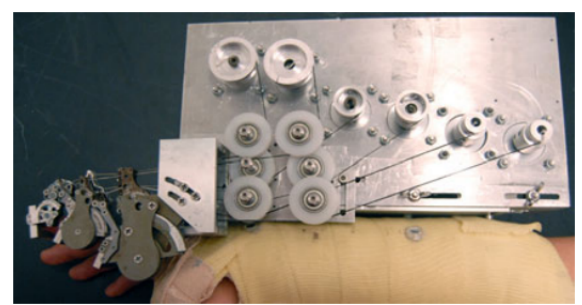

Pneumatic glove [3] on the left and cable driven robot finger [7] on the right

fingers totally individually, the thumb itself may require separate design due to its special function of opposition [14].

Other important property is number of separately controlled joints or the number of degrees of freedom. Pneumatic gloves control the extension of the whole fingers with a single actuator. The motor-cable systems may control each joint of each finger separately, this provides a great flexibility for the realized motion $[9,15]$. However, it is also possible control the last two joints [6] or all the three joints (common in the case of simple gloves) [4] together requiring fewer motors, but also resulting in less flexibility for the possible motions.

Our design utilizes the direct-drive approach, meaning that the motor are placed near the fingers and the force is applied directly on the middle phalanx (distal phalanx in the case of the thumb) through the robot body without any cable system. The movement of index, middle, ring and pinkie fingers are grouped, they are moved together using a single robotic arm with only three motors; so the number of necessary motors is highly reduced, still, the free motion of separate joints along the fingers is preserved. The thumb has its own robot finger with two motors, overall, this hand module has five degrees of freedom.

The module introduced in the article will be applied during the augmentation and further development of the REHAROB robotic physiotherapeutic equipment $[16,17]$ built in the Hungarian Medical Institute for Rehabilitation (OORI). The further development involves supplementing the REHAROB physiotherapeutic equipment with a hand module and making it capable of supporting active kinesiotherapy. The basic system assists the stressful tasks of physiotherapists engaged in the kinesiotherapy of patients who suffer from spastic hemiplegia called spasm in medical terminology - by applying industrial robots. The system consists of two industrial robots manufactured by ABB, a PC-based high-level controller, a motorised treatment seat, an object table, one hand- and several finger-orthoses and a custom-designed hand module. The system has two modes of operation, the first is the passive mode: in this mode, the therapist trains the robots to perform a given exercise. The robot is force-controlled during the training (in impedance mode), it records the trajectory of the movements, and then it is capable of replaying the saved trajectory, which means repeating the tasks (taught by the therapist) on the patient. The second is the active mode, where the exercises consist of five predetermined activities of daily living movements 


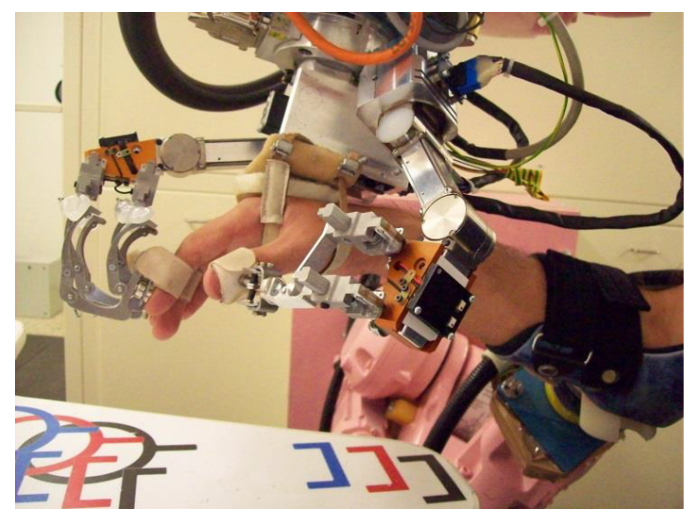

Figure 2

Structure of the REHAROB system (hand module, ABB robot arms)

(drinking, using zipper, wiping mouth with a sponge, using telephone, etc.).

The robot has a pre-written program for the sequence of movements, and performs the exercises on the basis thereof. The modified SDH (SCHUNK Gmbh. Dextrous Hand 2.0) hand module consists load cells, two robot fingers, and the mediator elements and finger orthoses mounted on the former. One finger is responsible for moving the thumb, the other is responsible for moving the index-, the middle- and the ring fingers. The little finger is not involved. Figure 2 shows the structure of the REHAROB system and the old hand module mounted onto the end of the IRB1600 robot mounted on the "ceiling", which implements the moving/rehabilitation of the hand.

\section{Design considerations}

The input data of the design (specifications) is based on the experiences obtained by clinical examinations [18] performed with the REHAROB robotic physiotherapeutic system shown in the Figure 2. The so-obtained design aspects are also based on the opinions of the dedicated medical team.

The proven features of the previous system are the following:

- Replaceable hand orthosis (standard sizes customizable for the patient).

- replaceable finger orthoses (orthoses with customizable sizes).

- Changeable left/right side (it is possible to perform physiotherapy both for the left and right side, ie. side change is possible).

- Adjustable orthoses.

- Absolute angle sensors installed in the joints.

- Applied admittance control. 
- Drive integrated in the central unit.

- Drive of each joint in stress-free condition (no self-locking transmissions).

The functions missing from the previous device and the its defects are summarized as follows:

- Overly complicated assembly.

- The removable connections are inadequate, the thumb finger module needs to be replaced for left-right side change. The unit moving the thumb needs to be replaced when changing the side, which entailed not only the mechanical reassembly of the modules, but also replacing the electrical wires. The connections were damaged often and the cables were broken when connected. The damages of the connections and cables resulted in shortcircuits.

- Few implemented degrees of freedom (2 controlled joints), under-actuated movement.

- Low performance (the folding-out torque of the joints is low: $1.4[\mathrm{Nm}]$ and $2.1[\mathrm{Nm}])$.

- The robot finger moving the three fingers (index-, middle- and ring finger) is asymmetric, its allocation is eccentric, so the module needs to be placed to the other side when side is changed.

- The modules - due to their geometrical sizes - are uncapable of encompassing the standard anatomical dimensions (60 percentiles for the female and 95 percentiles for the male hand).

- The modules - due to the physical dimensions of the load distributors mounted on them - are unable to implement gentle motions (grabbing and releasing objects).

The defects/deficiencies listed above made the development of a new construction necessary:

- Electrical wiring needs to be established within the finger.

- The degrees of freedom of the three-finger module has been increased: 3 driven joints, full closing has been solved.

- The three-finger module has been redesigned to be symmetrical: the module has been aligned to the centreline of the lower arm and of the hand. The module does not need to be replaced when changing the side, only the thumb-moving unit's position needs to be changed.

- The hand orthosis is replaceable: it can be removed and fixed with an locating pin.

- The unit moving the thumb is provided with a built-in electrical connection. No wiring is necessary when changing the side. 
- The load distributor is now part of the end of the three-finger robotic finger, so the encompassing size is minimized.

- A two-directional force measurement has been implemented at the end of both robot fingers.

- Built-in force measurement: the inner joint torques are now measured directly.

- The performance of the motors has been increased: application of 20[W] and $60[\mathrm{~W}]$ brushless direct current motors with electronic commutation.

- The overload protection of the measuring cells is implemented via mechanical collision: the built-in load cells are now protected against overload.

- Power electronics integrated in the joints (force measurement, position measurement, speed- and position control organized in the lower level).

\section{System Overview}

The structure of the modules consisting rotational joints with serial kinematics is as described below. Each rotational joint is actuated by harmonic drives, and directly-driving brushless DC motors (BLDC). The torques on the joints are sensed by small load sensors with a measuring limit of $100[\mathrm{~N}]$, which have been placed between the joints. Each force cells sense radial - ie. normal direction - forces, the torque on the joints can be calculated from the radial forces, in knowledge of the fixed geometrical distances between the joints. The occasional overload protection of the load cells is implemented by the mechanical collision of the housing. The force component perpendicular to the normal direction is sensed by micro force sensor placed at the end of the finger, which is capable of sensing compression and tension forces as well. The angle sensor of the joints is sensed by Hall type 14-Bit Programmable Magnetic Rotary Position Sensor. The joints are provided with integrated power electronics placed at the end of the BLDC motors, and receive the signals from the ADC SPI which is processing the absolute transducers and the force measurement cells. The AS5047 absolute encoder and the ADC122S101 ADC communicate with the dsPIC33EP512GM304 micro controller via two independent SPI lines. The controlling of the brushless motors is performed by the DRV8308 Brushless DC Motor Controller. The integrated power electronics panels use a full duplex RS-422 communication line to communicate with the communication distribution unit which is connected to the higher-level controller. The distributor unit receives the measured data of the joints (torque/forces, absolute positions, current rpms), and sends the setpoint calculated by the controller (speed reference) and the list of the related controlling parameters (values of the PID speed regulator's parameters). RS-422 hardware setup was applied due to the symmetrical and differential structure, in order to reduce sensitivity to communication disturbances. The slave units are scheduled by the synchronizing signal coming from the master, so the scheduling of the controller can be perpetuated. The communication jitter resulting from 
the communication of the system can be minimized. The power electronics panels integrated in the joints are powered by a 24[VDC] power supply, which also powers the final drive of the brushless motor and the DC-DC switching converter which generates the inner power supply required for the operation. The power electronics panel is located at the end of the motor, and an FFC connector links it to the inner communication PCB located in the joints of the robot finger. The signals between the rotation joints - including the RS-422 level-shifted separated $\mathrm{RX}$, TX communication signals of the rotation joints, the communication lines of the absolute angle sensor of the drive and the communication lines of the ADC SPI which is processing the load cell - are forwarded by the flexible printed circuit board. The flexible PCB is rolled around the joint to ensure free movement, so its length can change during rotation. The absolute encoder IC is placed on this circuit board as well. The aluminium console of the HAL IC is placed above the IC, fixed to the moving part of the joint with two bolts, so the bipolar magnet placed in the console is rotated together with the joint.

The power supply of the power electronics modules is solved within the joint by using flexible silicone cables (Stäubli FlexiVolt-E), since the guide strip's crosssection required due to the total power load of the electrical motors and the space available inside the joint does not allow forwarding the required power via a flexible circuit board. The flexible silicone cables are located above the flexible circuit board, their position and guiding are secured by the aluminium consoles of the HAL IC, and the cables are connected to the distributor panels in the joints. The inverse kinematic (mathematical) module implementing the robot finger's movement, as well as the admittance controller implementing the trajectory recording and power control are implemented in the higher-level controller. Real-time calculation capacity can be ensured easier and even the algorithm implementation is also faster using a PC-based architecture and a real-time operating system, than using an embedded device. The parameters of the admittance controller (required strength, attenuation) can be adjusted during the physiotherapy, so that the robot finger can achieve the desired results. Cascade controlling has been implemented in the joints, while speed regulation has been applied in each motor - in addition to subordinated voltage regulation and super-ordinated position regulation. The admittance controller provides position setpoint to the joints of the robot finger, the position trajectory determines the reaction force of the module, so the prescribed torque can be interpreted as a position setpoint within the regulatory chain. The prescribed position is generated by the motion of the circular spline of the harmonic drive, so the feedback of the position regulation is implemented on the folding-out part of the transmission, and so a dead time element is created in the regulatory chain, which has a relatively large time constant. This is the amount of time that needs to pass in the system to have the effect of the input appear on the output.

Figure 3 shows the flexible PCB rolled up in the joint (its 3D-printed test version) and the flexible silicone cables. The image shows the external final position of the joint. The length of the flexible cables is changing during the rotation of the joint, because the cables are not rotating along the same curve due to their diameter (they are located abreast). Their length change inside the housing is allowed by 


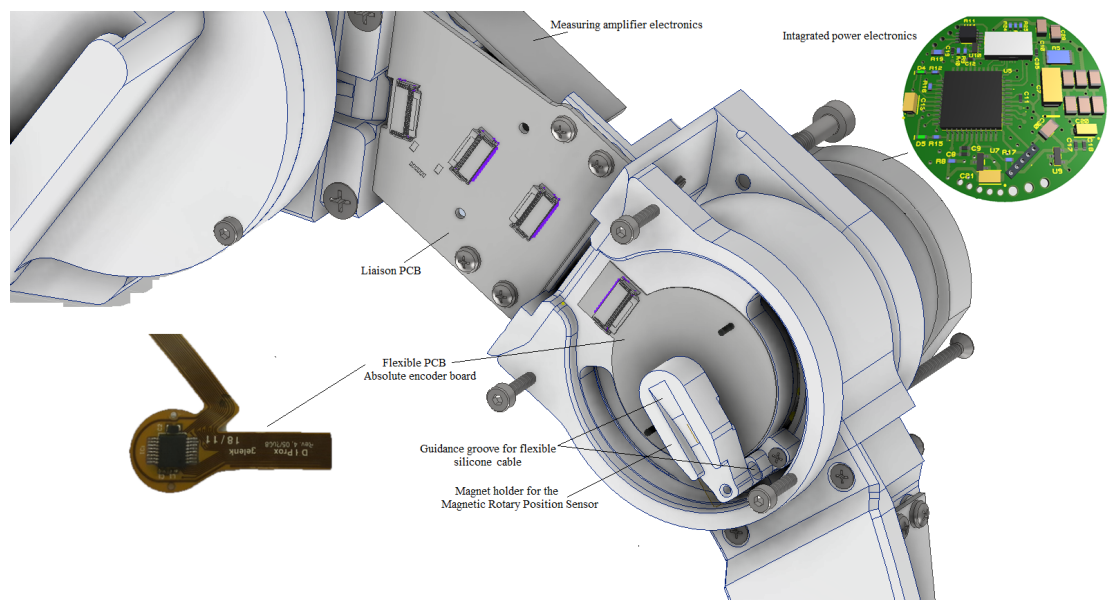

Figure 3

The second joint of the robotic hand with the integrated electronics and flexible PCB

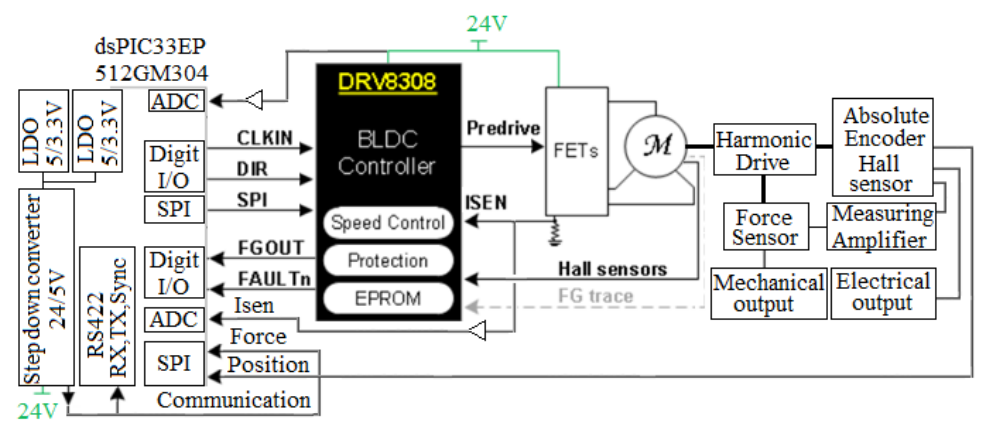

Figure 4

Schematics of the actuation hardware 
the free movement of the cables. The inner size and shape of the grooves on the housing was established according to the cables' bend radius. The flexible cables are connected to the inner circuit boards that connect the joints. Figure 4 shows the integrated power electronics schematic.

\section{Mechanical Design}

The rehabilitation device serving therapeutic purposes has two components: a planar module 3DoF RRR, and another module with 2DoF. The robot finger with three degrees of freedom moves the index-, the middle and the ring fingers, using a load distributor placed above the fingers. The finger orthoses are connected to the load distributor via magnets. The robot finger with two degrees of freedom moves the thumb according to closing, along the plane (tilted in two angles). We have built a measuring system to determine the required plane; this system consists of two perpendicular cameras. We've obtained the spatial location of the motion's trajectory by using the markers fixed to the thumb, from the crosssection of the two cameras' perspectives and from the images recorded frameby-frame during the motion. The coordinate values of the points recorded by the cameras could be imported into a CAD system. The motion's trajectory results in a conical surface; this surface can be approximated well by moving along a plane tilted in the proper degree, by implementing two degrees of freedom. Grabbing with the thumb is ensured by the orthosis located on the distal phalanx bone. The motion occurring upon closing the finger is allowed by the joint provided with a bearing.

The robot finger with three degrees of freedom allows the full opening and closing of the hand, and the module encompasses the hand's anthropometric size range between 60 and 95 percentile size. Hence, the device is fitting for the rehabilitation of male and female patients as well. The rehabilitation device is fixed to

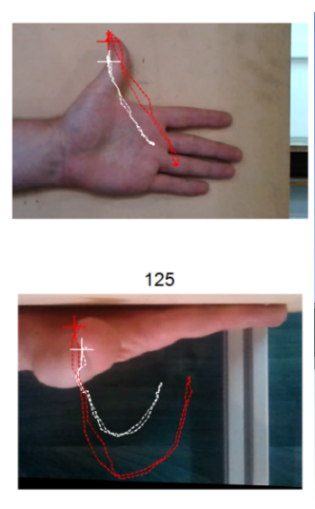

Thumb finger trajectories (measuring layout), and CAD model

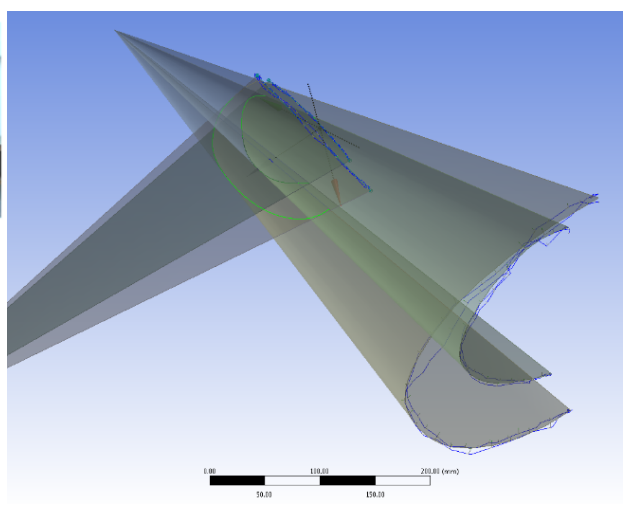

Figure 5 
the hand by a custom-designed hand orthosis, which can be fitted to the patient's hand in accordance with the size standards (Small, Medium, Large), while the orthosis ensures the hand's stable grasp by a spring prestressing. The design of the inner surface's curve follows the curve of the back of the hand. The rehabilitation device allows changing between the left- and the right hand, which allows the rehabilitation of both sides.

Two sizes of drives and two kinds of motors have been used in the robot finger. The smaller joints producing a torque of $4.8[\mathrm{Nm}]$, while the torque of the larger joints is $11[\mathrm{Nm}]$. The position is measured on the side of folding-out, directly in relation to the fixed pillow block (see Figure 3); this provides the feedback signal of the position control. The load cells integrated between each joint measure the reaction forces, the torques of the joints can be calculated in knowledge of the geometry. The implemented mechanical construction allows a rigid and wellcontrolled structure, which has a smaller loss and is much more accurate than the indirect cable-controlled tendon actuation. A two-component force meter (two load cell) has been installed at the end of the fingers where the orthoses are fixed, so in addition to the torque of the joints, we can even measure the force component perpendicular to the torque.

Figure 7 shows the 3D design of the rehabilitation device serving therapeutic purposes. The device and the UR-10 e-Series robot which moves the upper limb are linked by an adapter which is fitted to the flange-end of the robot. The image shows the T-nut-shaped fixing insert which allows interchangeability and implements the tilt angles of the $2 \mathrm{DoF}$ thumb module. It is clearly visible in the image that the thumb module is fixed in a different angle on the left side of the device than it is fixed in horizontal position on the right side, because of the side change. The module fixing T-nut insert is provided with a built-in electrical connection, which solves the module's electrical connection after a mechanical stabilisation when sides are changed. Side change is easily achieved by the telescopic indexing plungers. The finger orthoses on the patient's fingers are fixed to the rehabilitation device by using magnets.

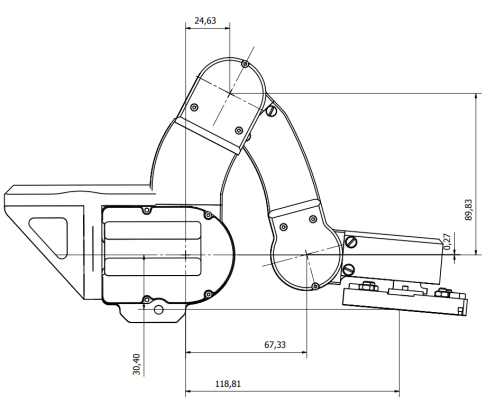

(a)

Fully opened (the hand is stretched)

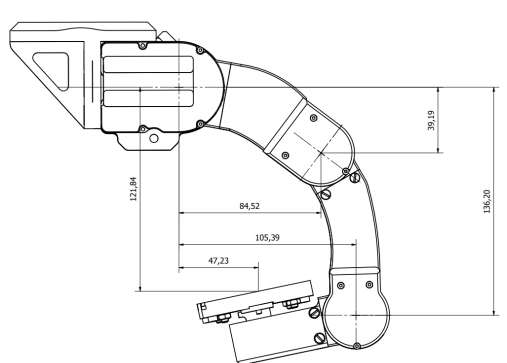

(b)

Fully closed (fists clenched)

Figure 6

The design of the direct drive robot hand 


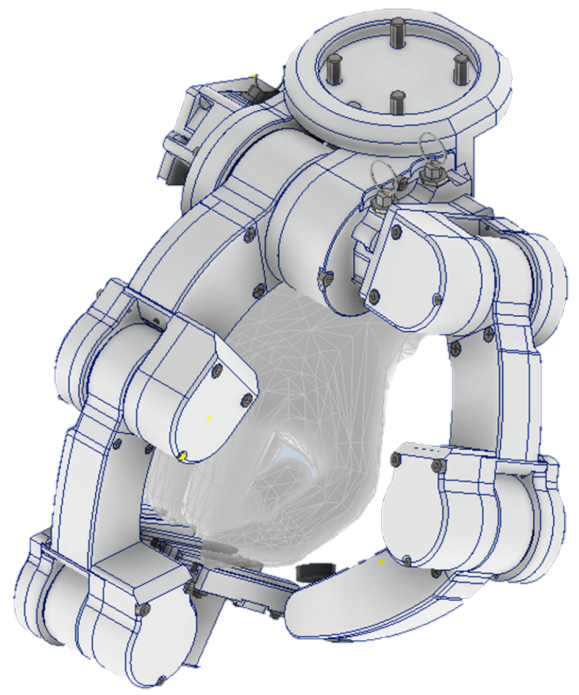

Figure 7

The proposed arrangement of the hand exoskeleton

\section{System modelling}

In this Section, the kinematics and dynamics of the proposed equipment is discussed.

\subsection{Kinematics}

The relative joint angles are denoted as $\theta_{1}, \theta_{2}$ and $\theta_{3}$, the end point coordinates are denoted by $x$ and $y$, while $\varphi$ is the absolute angle of the last segment. The mechanical model is shown in Figure 8.

The forward kinematics can be derived using basic geometry, the $\left(l_{1}, l_{2}, l_{3}\right)$ parameters are the lengths of the corresponding segments:

$$
\left[\begin{array}{l}
x \\
y \\
\varphi
\end{array}\right]=f\left(\theta_{1}, \theta_{2}, \theta_{3}\right)=\left[\begin{array}{c}
l_{1} \cos \left(\theta_{1}\right)+l_{2} \cos \left(\theta_{1}+\theta_{2}\right)+l_{3} \cos \left(\theta_{1}+\theta_{2}+\theta_{3}\right) \\
l_{1} \sin \left(\theta_{1}\right)+l_{2} \sin \left(\theta_{1}+\theta_{2}\right)+l_{3} \sin \left(\theta_{1}+\theta_{2}+\theta_{3}\right) \\
\theta_{1}+\theta_{2}+\theta_{3}
\end{array}\right] .
$$

The Jacobian matrix of the forward kinematics $f$ is: 


$$
\begin{aligned}
& \mathbf{J}=\left[\begin{array}{c}
-l_{1} \sin \left(\theta_{1}\right)-l_{2} \sin \left(\theta_{1}+\theta_{2}\right)-l_{3} \sin \left(\theta_{1}+\theta_{2}+\theta_{3}\right) \\
l_{1} \cos \left(\theta_{1}\right)+l_{2} \cos \left(\theta_{1}+\theta_{2}\right)+l_{3} \cos \left(\theta_{1}+\theta_{2}+\theta_{3}\right) \\
1
\end{array}\right. \\
& -l_{2} \sin \left(\theta_{1}+\theta_{2}\right)-l_{3} \sin \left(\theta_{1}+\theta_{2}+\theta_{3}\right) \quad-l_{3} \sin \left(\theta_{1}+\theta_{2}+\theta_{3}\right) \\
& l_{2} \cos \left(\theta_{1}+\theta_{2}\right)+l_{3} \cos \left(\theta_{1}+\theta_{2}+\theta_{3}\right) \quad l_{3} \cos \left(\theta_{1}+\theta_{2}+\theta_{3}\right) \\
& 1 \\
& 1
\end{aligned}
$$

The determinant of the Jacobian matrix $\mathbf{J}$ is:

$$
\operatorname{det} \mathbf{J}=l_{1} l_{2} \sin \theta_{2},
$$

so the singular positions are $\theta_{2}=0, \pm \pi \operatorname{rad}$ or $0^{\circ}, \pm 180^{\circ}$, which means that the middle segment is straight with respect to the first segment. This position should be avoided during the control, because the inverse kinematics calculation is non-unique here, this requirement is naturally fulfilled by the planned motions (human-hand physiotherapy), so no further treatment is necessary. The inverse

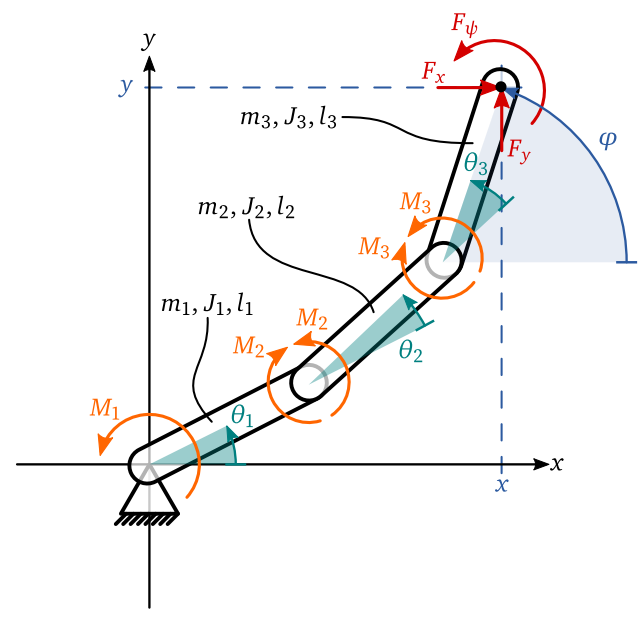

Figure 8

Mechanical model of the $3 \mathrm{DoF}$ exoskeleton finger 


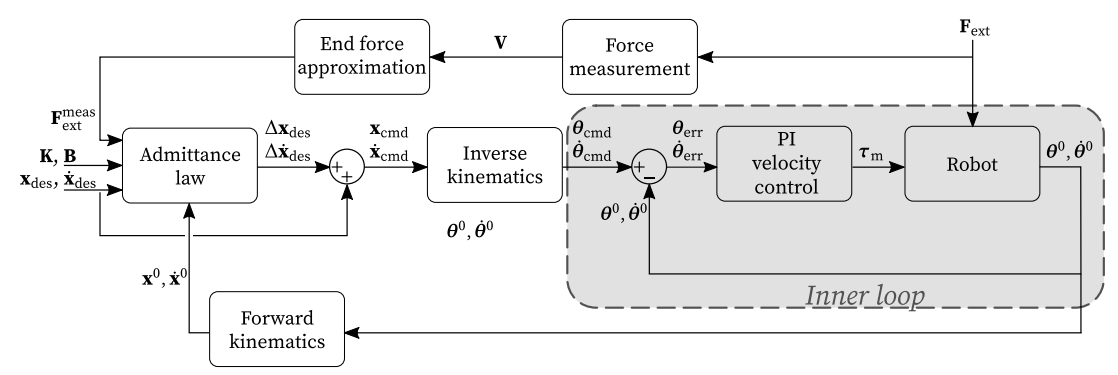

Figure 9

Block diagram of the admittance control

kinematics can be described locally by the inverse Jacobian matrix:

$$
\begin{aligned}
& \mathbf{J}^{-1}\left(\theta_{1}, \theta_{2}, \theta_{3}\right)= \\
& {\left[\begin{array}{ccc}
\frac{\cos \left(\theta_{1}+\theta_{2}\right)}{l_{1} \sin \left(\theta_{2}\right)} & \frac{\sin \left(\theta_{1}+\theta_{2}\right)}{l_{1} \sin \left(\theta_{2}\right)} & \frac{l_{3} \sin \left(\theta_{3}\right)}{l_{1} \sin \left(\theta_{2}\right)} \\
-\frac{l_{1} \cos \left(\theta_{1}\right)+l_{2} \cos \left(\theta_{1}+\theta_{2}\right)}{l_{1} l_{2} \sin \left(\theta_{2}\right)} & -\frac{l_{1} \sin \left(\theta_{1}\right)+l_{2} \sin \left(\theta_{1}+\theta_{2}\right)}{l_{1} l_{2} \sin \left(\theta_{2}\right)} & -\frac{l_{3}\left(l_{1} \sin \left(\theta_{2}+\theta_{3}\right)+l_{2} \sin \left(\theta_{3}\right)\right)}{l_{1} l_{2} \sin \left(\theta_{2}\right)} \\
\frac{\cos \left(\theta_{1}\right)}{l_{2} \sin \left(\theta_{2}\right)} & \frac{\sin \left(\theta_{1}\right)}{l_{2} \sin \left(\theta_{2}\right)} & \frac{l_{2}+\frac{l_{3} \sin \left(\theta_{3}\right)}{\tan \left(\theta_{2}\right)}+l_{3} \cos \left(\theta_{3}\right)}{l_{2}}
\end{array}\right]}
\end{aligned}
$$

\subsection{Control}

A cascade controller is designed for the robot fingers, the inner (fast) loop is velocity controller separately for each motor. The outer (slow) loop is a PC based admittance controller which provides the reference signal for the inner loop, the high level controller is responsible for the compliant behavior.

The deviation $\Delta \mathbf{x}_{\text {des }}$ from the reference trajectory $\mathbf{x}_{\text {des }}$ can be obtained by the admittance law:

$$
\Delta \mathbf{x}_{\mathrm{des}}=\mathbf{K}^{-1}\left(\mathbf{F}_{\mathrm{ext}}-\mathbf{B} \dot{\mathbf{x}}\right),
$$

this term causes the compliant behavior of the end effector. The $\mathbf{K}$ and $\mathbf{B}$ matrices are the corresponding stiffness and damping matrices, respectively; the compliance can be tuned through these parameters.

The end effector velocity is calculated with the Jacobian $\mathbf{J}\left(\theta_{0}\right)$ as $\dot{\mathbf{x}}=\mathbf{J}\left(\theta_{0}\right) \dot{\boldsymbol{\theta}}$. The external force is obtained from the measured force signals $\mathbf{V}$ by the transformation $\mathbf{F}_{\mathrm{ext}}^{\text {meas }}=\mathbf{T}\left(\theta_{0}\right) \mathbf{V}$.

The inverse kinematics is approximated by the inverse Jacobian $\mathbf{J}^{-1}\left(\theta_{0}\right)$ as

$$
\Delta \theta=\mathbf{J}^{-1}\left(\theta_{0}\right) \Delta \mathbf{x}
$$


where $\Delta \mathbf{x}=\mathbf{x}_{\mathrm{cmd}}-\mathbf{x}_{0}$ is the difference of command position $\mathbf{x}_{\mathrm{cmd}}$ and current position of the end effector $\mathbf{x}_{0}$.

Finally, the deviation $\Delta \theta$ and the reference joint angles $\theta_{\mathrm{cmd}}$ can be expressed as

$$
\begin{aligned}
\Delta \theta & =\mathbf{J}^{-1}\left(\theta_{0}\right) \mathbf{K}^{-1}\left(\mathbf{T}\left(\theta_{0}\right) \mathbf{V}-\mathbf{B J}\left(\theta_{0}\right) \dot{\theta}\right), \\
\theta_{\mathrm{cmd}} & =\theta_{0}+\Delta \theta,
\end{aligned}
$$

the reference joint angles are used in the inner control loop.

The equivalent torsional stiffness matrix with respect to the measured forces $\mathbf{V}$ can be defined as:

$$
\mathbf{K}_{\text {eqv }}^{-1}=\mathbf{J}^{-1}\left(\theta_{0}\right) \mathbf{K}^{-1} \mathbf{T}\left(\theta_{0}\right),
$$

This may be used in the inner control loop to improve the performance of the overall system with doing more calculations in the fast inner control loop.

\subsection{Simulation}

Beside the kinematic model, the dynamical model was also developed in order to test the performance of the system via numerical simulations. The simulations were carried out by using the Python programming language with the scientific libraries NumPy, SciPy, SymPy and Matplotlib. The setup was the following, at $t=0 \mathrm{~s}$ start the simulation at a realistic position of the robot, at $t=1 \mathrm{~s}$ the end effector is pushed by an $F=10 \mathrm{~N}$ force either in the $\mathrm{x}$ or in the y direction until the end of the simulation at $t=5 \mathrm{~s}$.

Figure 10 shows the initial configuration of the robot at the beginning of the simulation and at the final configuration at the end of the simulation. Figures 11 and 12 contain the joint angles and end point coordinates during the simulation, respectively. Minor error can be seen in the end point coordinates, when the external force is in the $\mathrm{y}$ direction there is a minimal change in the $\mathrm{x}$ direction as well. This is related to two reason: one is the approximated inverse kinematics and the relatively large position change, the other is the change in the static force/moment equilibrium caused by the external force. This small error is fully acceptable in the case of human hand physiotherapy; overall, the system behaves as expected.

\section{Conclusion}

We developed a hand exoskeleton for robotic post-stroke rehabilitation. In contrast to the different structures that exist, we proposed a unique direct drive solution with integrated force measurement.

Advantages of the proposed exoskeleton:

- Compact, lightweight design 

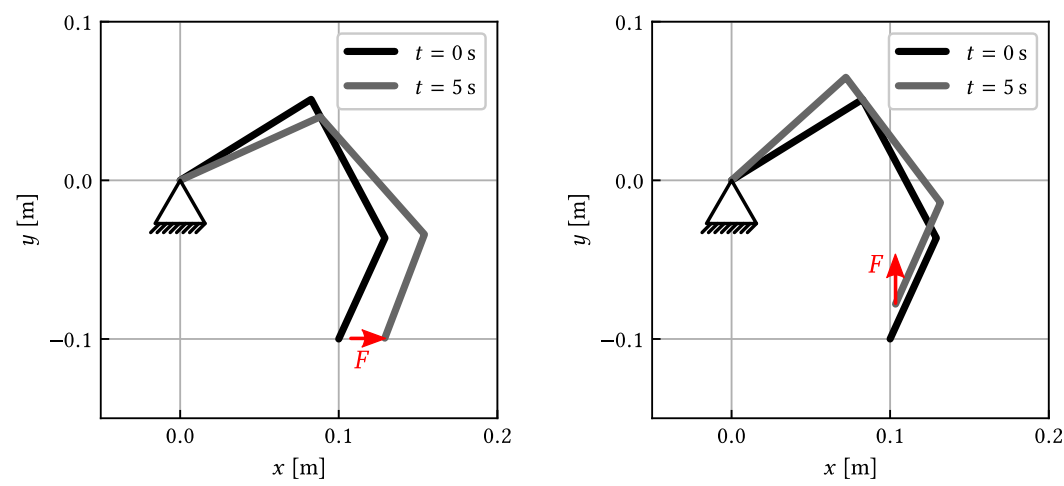

Figure 10

The robot arm configuration at the beginning of the simulation $t=0 \mathrm{~s}$ and at the end of the simulation $t=5 \mathrm{~s}$; the external force $F$ is in the $x$ direction (on the left) and in the $y$ direction (on the right)
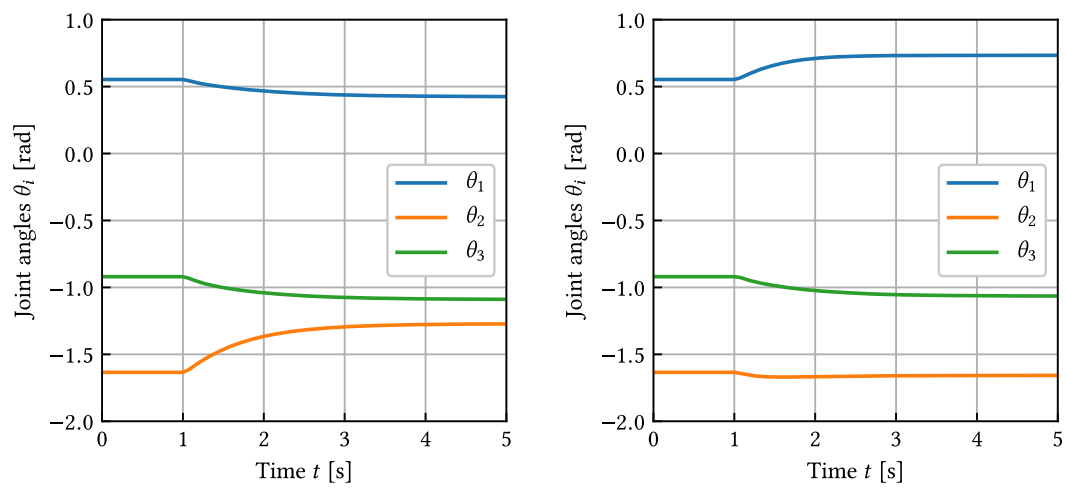

Figure 11

Time evolution of the relative joint angles, the external force $F$ is in the $x$ direction (on the left) and in the $y$ direction (on the right)
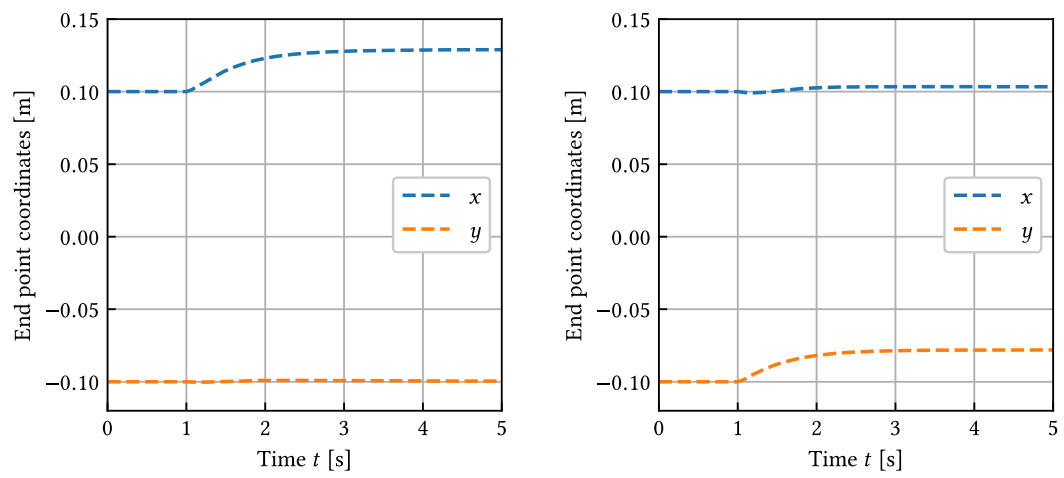

Figure 12

Time evolution of end effector coordinates, the external force $F$ is in the $x$ direction (on the left) and in the $y$ direction (on the right) 
- Changeable left/right side (it is possible to perform physiotherapy both for the left and right side, i.e. side change is possible) tegration to lower limb rehabilitation systems

- Wide anatomical size coverage

- High effort

- Built-in force measurement: the inner joint torques are measured directly

- High performance motors

The clinical trials will be realized in the next phase of the research, when the exoskeleton are completed and after the assembly are analyzed experimentally.

\section{Acknowledgment}

This work has been implemented with the support provided from the National Research, Development and Innovation Fund of Hungary, financed under the GINOP-2.3.3-15-2016-00032 funding scheme. Péter Galambos thankfully acknowledge the financial support of this work by the Hungarian State and the European Union under the EFOP-3.6.1-16-2016-00010 project.

Also, we would like to express our appreciation to assistant professor Dr. Bálint Magyar for the implementation of the measurements (BME, Faculty of Mechanical Engineering Department of Applied Mechanics).

\section{References}

[1] L. Connelly, Y. Jia, M. L. Toro, M. E. Stoykov, R. V. Kenyon, and D. G. Kamper. A pneumatic glove and immersive virtual reality environment for hand rehabilitative training after stroke. IEEE Transactions on Neural Systems and Rehabilitation Engineering, 18(5):551-559, 2010.

[2] Y. Kim, S. S. Cheng, A. Ecins, C. Fermüller, K. P. Westlake, and J. P. Desai. Towards a robotic hand rehabilitation exoskeleton for stroke therapy. In ASME 2014 Dynamic Systems and Control Conference. American Society of Mechanical Engineers Digital Collection, 2014.

[3] H. K. Yap, J. H. Lim, J. C. H. Goh, and C.-H. Yeow. Design of a soft robotic glove for hand rehabilitation of stroke patients with clenched fist deformity using inflatable plastic actuators. Journal of Medical Devices, 10(4), 2016.

[4] A. Stilli, A. Cremoni, M. Bianchi, A. Ridolfi, F. Gerii, F. Vannetti, H. A. Wurdemann, B. Allotta, and K. Althoefer. Airexglove-a novel pneumatic exoskeleton glove for adaptive hand rehabilitation in post-stroke patients. In 2018 IEEE International Conference on Soft Robotics (RoboSoft), pages 579-584. IEEE, 2018.

[5] B. Wang, A. McDaid, M. Biglari-Abhari, and K. C. Aw. Design and development of a glove for post-stroke hand rehabilitation. In 2017 IEEE International Conference on Advanced Intelligent Mechatronics (AIM), pages 1047-1051. IEEE, 2017.

[6] M. Cempini, S. M. M. De Rossi, T. Lenzi, M. Cortese, F. Giovacchini, N. Vitiello, and M. C. Carrozza. Kinematics and design of a portable and 
wearable exoskeleton for hand rehabilitation. In 2013 IEEE 13th International Conference on Rehabilitation Robotics (ICORR), pages 1-6. IEEE, 2013.

[7] C. L. Jones, F. Wang, R. Morrison, N. Sarkar, and D. G. Kamper. Design and development of the cable actuated finger exoskeleton for hand rehabilitation following stroke. IEEE/ASME Transactions on Mechatronics, 19(1):131-140, 2012.

[8] T. Worsnopp, M. Peshkin, J. Colgate, and D. Kamper. An actuated finger exoskeleton for hand rehabilitation following stroke. In 2007 IEEE 10th international conference on rehabilitation robotics, pages 896-901. IEEE, 2007.

[9] A. Chiri, N. Vitiello, F. Giovacchini, S. Roccella, F. Vecchi, and M. C. Carrozza. Mechatronic design and characterization of the index finger module of a hand exoskeleton for post-stroke rehabilitation. IEEE/ASmE Transactions on mechatronics, 17(5):884-894, 2011.

[10] M. A. Delph, S. A. Fischer, P. W. Gauthier, C. H. M. Luna, E. A. Clancy, and G. S. Fischer. A soft robotic exomusculature glove with integrated semg sensing for hand rehabilitation. In 2013 IEEE 13th International Conference on Rehabilitation Robotics (ICORR), pages 1-7. IEEE, 2013.

[11] S. Mohamaddan and T. Komeda. Wire-driven mechanism for finger rehabilitation device. In 2010 IEEE International Conference on Mechatronics and Automation, pages 1015-1018. IEEE, 2010.

[12] C. L. Jones, F. Wang, C. Osswald, X. Kang, N. Sarkar, and D. G. Kamper. Control and kinematic performance analysis of an actuated finger exoskeleton for hand rehabilitation following stroke. In $20103 \mathrm{rd}$ IEEE RAS \& EMBS International Conference on Biomedical Robotics and Biomechatronics, pages 282-287. IEEE, 2010.

[13] Y. Fu, Q. Zhang, F. Zhang, and Z. Gan. Design and development of a hand rehabilitation robot for patient-cooperative therapy following stroke. In 2011 IEEE International Conference on Mechatronics and Automation, pages 112-117. IEEE, 2011.

[14] F. Wang, M. Shastri, C. L. Jones, V. Gupta, C. Osswald, X. Kang, D. G. Kamper, and N. Sarkar. Design and control of an actuated thumb exoskeleton for hand rehabilitation following stroke. In 2011 IEEE International Conference on Robotics and Automation, pages 3688-3693. IEEE, 2011.

[15] C. L. Jones, F. Wang, R. Morrison, N. Sarkar, and D. G. Kamper. Design and Development of the Cable Actuated Finger Exoskeleton for Hand Rehabilitation Following Stroke. IEEE/ASME Transactions on Mechatronics, 19(1):131-140, February 2014. Conference Name: IEEE/ASME Transactions on Mechatronics.

[16] G. Fazekas, M. Horvath, and A. Toth. A novel robot training system designed to supplement upper limb physiotherapy of patients with spastic hemiparesis. International Journal of Rehabilitation Research, 29(3):251254, 2006.

[17] G. Fazekas, M. Horvath, T. Troznai, and A. Toth. Robot-mediated upper limb physiotherapy for patients with spastic hemiparesis: a preliminary 
study. Journal of rehabilitation medicine, 39(7):580-582, 2007.

[18] O. Peter, I. Tavaszi, A. Toth, and G. Fazekas. Exercising daily living activities in robot-mediated therapy. Journal of physical therapy science, 29(5):854-858, 2017. 